OPEN ACCESS

Edited by:

Gustavo Henrique Goldman,

University of São Paulo, Brazil

Reviewed by:

Peter Hore,

University of Oxford, United Kingdom Elisa Michelini,

University of Bologna, Italy

${ }^{*}$ Correspondence:

Fusheng Chen

chenfs@mail.hzau.edu.cn

Specialty section:

This article was submitted to Fungi and Their Interactions,

a section of the journal

Frontiers in Microbiology

Received: 13 January 2020

Accepted: 04 May 2020

Published: 19 June 2020

Citation:

Zhou H, Yang S and Chen F

(2020) The Magnetic Receptor

of Monascus ruber M7: Gene Clone and lts Heterologous Expression

in Escherichia coli.

Front. Microbiol. 11:1112. doi: 10.3389/fmicb.2020.01112

\section{The Magnetic Receptor of Monascus ruber M7: Gene Clone and Its Heterologous Expression in Escherichia coli}

\author{
Hongyi Zhou ${ }^{1,2}$, Shuyan Yang ${ }^{1,2}$ and Fusheng Chen ${ }^{1,2 *}$ \\ 1 Hubei International Scientific and Technological Cooperation Base of Traditional Fermented Foods, Huazhong Agricultural \\ University, Wuhan, China, ${ }^{2}$ College of Food Science and Technology, Huazhong Agricultural University, Wuhan, China
}

It is well known that many organisms can perceive the magnetic field (MF), including the geomagnetic field, but how to feel MF is unclear. Recently, a study has claimed that a biological compass, namely a complex of the magnetic receptor (MagR) and blue light (BL) receptor (cryptochrome), has been found in Homo sapiens, Drosophila melanogaster, and Danaus plexippus, which may bring some new ideas to explore the mechanism of biomagnetism. Monascus spp. are edible filamentous fungi that can produce abundant beneficial secondary metabolites and have been used to produce food colorants for nearly 2000 years in the world, especially in China, Japan, and Korea. In this work, we firstly treated M. ruber M7 by BL (500 lux,465-467 nm), MF (5, 10, $30 \mathrm{mT})$, and the combination of MF and $\mathrm{BL}(\mathrm{MF}-\mathrm{BL})$, respectively. The results revealed that, compared with the control (CK, neither BL nor MF), the MF alone had no effect on the growth and morphological characteristics of $\mathrm{M} 7$, but $\mathrm{BL}$ made the colonial diameters only $66.7 \%$ of CK's and inhibited the formation of cleistothecia. Under MF-BL, the colony diameters were still $66.7 \%$ of CK's, but the colonial growth and cleistothecia production inhibited by $\mathrm{BL}$ were partially restored. Then, we have found that the magR gene widely exists in the genomes of animals, plants, and microorganisms, and we have also discovered a magR gene in the M7 genome, hereinafter referred to $m r-m a g R$. Finally, the full-length cDNA of mr-magR was successfully cloned and expressed in Escherichia coll BL21 (DE3), and the Mr-MagR protein was purified by a Ni ${ }^{+}-\mathrm{NTA}$ column and identified by Western blot. These results have laid a foundation for further investigation on the relationship between Mr-MagR and BL receptor(s) that might exist in M7. According to a literature search, it is the first time to report magR in filamentous fungi.

Keywords: Monascus ruber, magnetic receptor, blue light, heterologous expression, Western blot

\section{INTRODUCTION}

Many organisms, including animals (Cain et al., 2005; Zhan et al., 2011; O'Neill, 2013), plants (Garcia and Arza, 2001; Zhadin, 2001; Anand et al., 2012), and microorganisms (Blakemore, 1975; Frankel et al., 1979; Faivre and Schuler, 2008; Rakoczy et al., 2016) can perceive the magnetic field (MF), including the geomagnetic field. To explain the phenomenon of biological MF perception, 
several hypotheses and models have been proposed (Frankel and Blakemore, 1980; Liboff et al., 1987; Ritz et al., 2000; Johnsen and Lohmann, 2005; Wiltschko, 2012). Among them, the magnetite-based model and the radical-pairreaction-based chemical model (RCM) are probably the most well-researched hypotheses so far (Ritz et al., 2004; Mouritsen and Ritz, 2005; Maeda et al., 2008; Rodgers and Hore, 2009; Mouritsen and Hore, 2012; Giachello et al., 2016; Hore and Mouritsen, 2016; Nordmann et al., 2017; Sherrard et al., 2018). The RCM means that organisms can perceive MF signals through the quantum spin dynamics of a radical-pair reaction produced from the activation of cryptochrome (Cry), a kind of flavoprotein, also known as a blue light (BL) receptor in various organisms (Hore and Mouritsen, 2016), and this hypothesis has also been verified in cry-deficient Drosophila melanogaster who lose the response to MF (Gegear et al., 2008). However, the RCM fails to explain how D. melanogaster can sense the changes in the MF intensity and orientation (Hore and Mouritsen, 2016).

Recently Qin et al. (2016) claimed that they have found a homologous protein of the bacterial iron-sulfur cluster (ISC) assembly ISCA1, called a magnetic receptor (MagR), in Homo sapiens, D. melanogaster, and other species, which can combine with Cry to form a protein complex (MagR-Cry), namely a biological compass, to sense the changes in direction of MF, thus providing a new clue to explore the mechanism of biologically sensing MF. The ISC protein widely exists in Homo sapiens, animals, plants, and microorganisms (Wang H. X. et al., 2016) and possesses many biological functions, such as maintaining mitochondrial stability (Jensen and Culotta, 2000; Kaut et al., 2000; Pelzer et al., 2000), regulating iron ion binding and iron homeostasis in Saccharomyces cerevisiae (Krebs et al., 2001; Ding et al., 2005), and adjusting the circadian rhythm of animals (Kosmidis et al., 2011; Mandilaras and Missirlis, 2012). However, up to now, the role of ISC magneto-sensing in filamentous fungi has not been reported.

Monascus spp., which are edible filamentous fungi and can produce abundant secondary metabolites (SMs), such as Monascus pigments, monacolin $\mathrm{K}$, citrinin, and so on (Chen et al., 2017), have been used for nearly 2,000 years in the world, especially in China, Japan, and other Asian countries (Chen et al., 2015). Previous studies have revealed that almost all fungi can sense and receive light signals through light receptors, such as green-light receptors, red-light receptors, and BL receptors (Schumacher, 2017). Among them, the BL receptor, Cry is the best-studied light receptor in fungi until now (Casas-Flores and Herrera-Estrella, 2016).

Recently, our and other research groups have discovered that BL and MF have significant effects on SMs production of Monascus spp. (Zhang et al., 2015; Wang L. et al., 2016; Wan et al., 2017). So Monascus spp. may exist a protein complex, such as MagR-Cry, to respond to the magnetic and light signals, and we have also discovered a $\operatorname{magR}$ gene in $M$. ruber $M$ 7 , hereinafter referred to $m r-m a g R$, but we did not find any homologous gene similar to cry or other BL receptor genes in the M7 genome that commonly appear in other fungi. Thus, we put forward a hypothesis that there might be an unknown
$\mathrm{BL}$ receptor in $M$. ruber $\mathrm{M7}$, namely $\mathrm{Mr}-\mathrm{BLR}$, to form a $\mathrm{Mr}$ MagR-BLR complex to sense MF and BL signals. In order to explore this hypothesis, we firstly investigated the effects of $\mathrm{BL}$, MF, and a combination of MF and BL (MF-BL) on the growth and morphological characteristics of $\mathrm{M} 7$, respectively, and found that MF-BL had the most significant effects on the M7 strain. Then, we searched the genomes of animals, plants, and microorganisms based on MagR of D. melanogaster (dMagR), and summarized a total of 73 proteins' amino acid (AA) sequences with a dMagR similarity greater than $55 \%$ to construct a phylogenetic tree and analyzed AA sequences of Mr-MagR. After that, the full-length cDNA sequence of $m r$-magR was cloned, analyzed, and expressed in Escherichia coli, and Mr-MagR protein was purified by a $\mathrm{Ni}^{+}$-NTA column and identified by Western blot.

The abovementioned results have laid a foundation to seek Mr-BLR and investigate the relationship between $\mathrm{Mr}$ MagR and Mr-BLR, which might exist in M7. According to a literature search, it is the first time to report the $\operatorname{mag} R$ gene in filamentous fungi.

\section{MATERIALS AND METHODS}

\section{Strains and Plasmids}

M. ruber M7 (CCAM 070120, Culture Collection of State Key Laboratory of Agricultural Microbiology, Wuhan, China) is stored in our laboratory (Chen and $\mathrm{Hu}, 2005$ ). The pET-28a plasmid is deposited in our laboratory, too. E. coli $[\mathrm{DH} 5 \alpha$ and BL21(DE3)] competent cells were purchased from TransGen Biotech Co., Ltd. (Beijing, China) and cultured in Luria-Bertani (LB) medium supplemented with $50 \mu \mathrm{g} / \mathrm{mL}$ kanamycin or ampicillin when required.

\section{Effects of MF, BL, and MF-BL on Morphologies of $M$. ruber M7}

In order to investigate the effects of MF, BL, and MF-BL on M7, we have built a device of coupling light and MF (Figure 1). In the device, two permanent magnets are clamped by fixtures to form a magnet pair, and the magnetic flux densities between the two magnets can be controlled by adjusting the distance of the magnets; meanwhile, a light-emitting diode (LED) panel is placed on the bottom of the device, and the LED's BL (465$467 \mathrm{~nm}$ ) and its intensities can be monitored by the control system, including a power transformer, an LED color converter, and a brightness regulator of the sliding rheostat (Figure 1). The magnetic densities are detected with a Gauss meter (SJ700, Senjie Technology Co., Ltd., Guilin, China), and the BL intensities are measured by a light meter (VICTOR 1010A/D, Shengli Gao Electronic Technology Co., Ltd., Shenzhen, China). We confirmed that the intensity of MF in this study did not change the intensity of BL emitted by the LED.

After the M7 strain is cultured on potato dextrose agar (PDA) for 15 days at $28^{\circ} \mathrm{C}$, its spores are harvested and adjusted to $1.0 \times 10^{6}$ spores $/ \mathrm{mL}$ with sterile water. Then $100 \mu \mathrm{L}$ spore suspension is spread and inoculated onto PDA. Finally, PDA plates are put in the middle between the two permanent magnets (Figure 1) and incubated at $28^{\circ} \mathrm{C}$ for 10 days alone under 5,10 , 


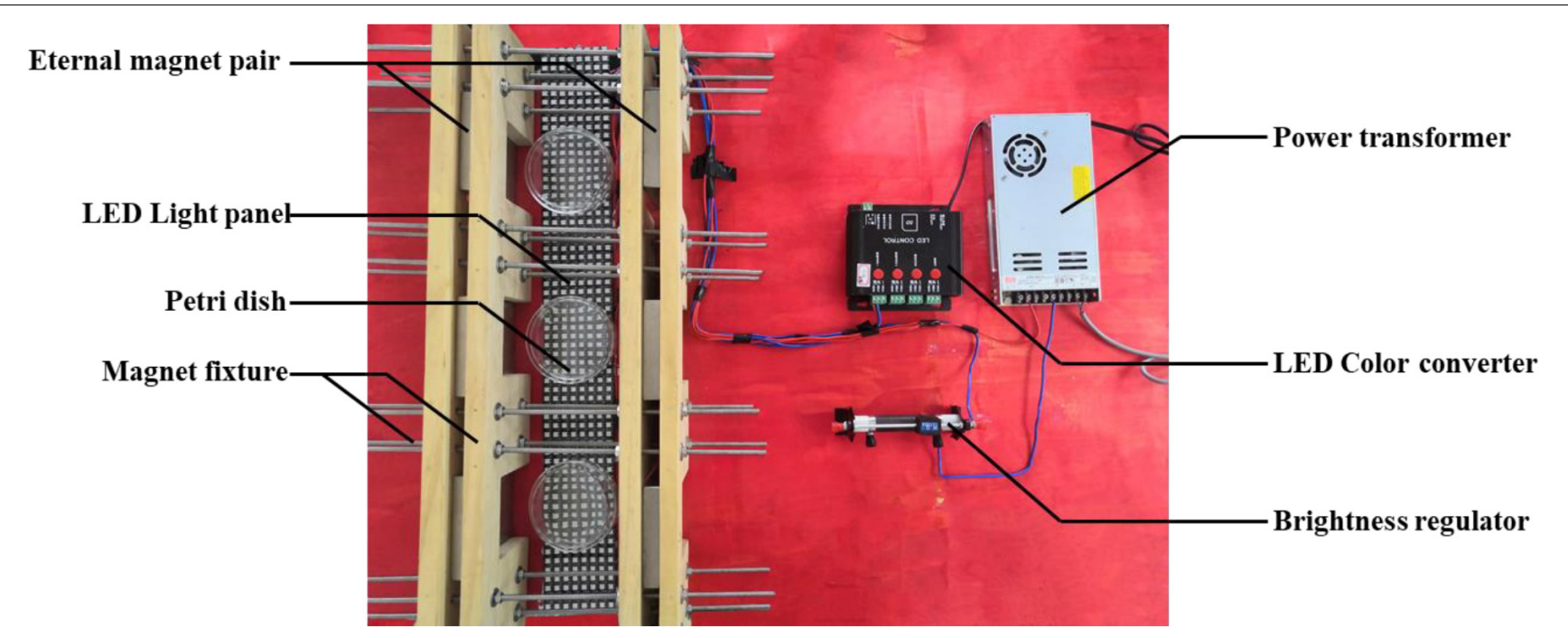

FIGURE 1 | The photo of the device coupling lights and magnetic fields.

and $30 \mathrm{mT}$ MF by turning off $\mathrm{BL}, 500$ lux $\mathrm{BL}$ by removing MF, and MF-BL by turning on the $\mathrm{BL}$, respectively, to investigate the effects of $\mathrm{MF}, \mathrm{BL}$, and $\mathrm{MF}-\mathrm{BL}$ on the colonial and microscopic morphologies of $M$. ruber M7 compared with the control (CK, neither BL nor MF).

Three parallel experimental groups are conduct for each condition. Colony diameter are defined as: Take two straight lines on the colony pass through the center of the colony, take the average value of the two straight lines. Calculate the standard deviation of the colony diameter of three parallel experiments.

\section{Bioinformatics Analysis of Mr-MagR in M. ruber M7 \\ Phylogenetic Analysis of Mr-MagR}

Based on AA sequences of the MagR (D. melanogaster), we used NCBI-BLASTP to search the genomes of Homo sapiens; animals; plants; filamentous fungi, including Monascus spp., Saccharomyces cerevisiae, Actinomyces spp. and bacteria to find the MagR protein. MagRs, which are homologically similar with dMagR higher than 55\%, were selected to draw a phylogenetic tree by MEGA 7 (Kumar et al., 2016) and iTOL 4.0 with the maximum likelihood method (Letunic and Bork, 2019). Then several bioinformatics tools, including NCBI-CDD (Marchler-Bauer et al., 2016), Pfam (El-Gebali et al., 2018), and DNAMAN software, were applied to analyze the conserved domains and conserved AA sites of MagR proteins.

\section{The Structure and Property Analyses of Mr-MagR}

Softberry $^{1}$ and NCBI ORF-Finder were used to compare and analyze the numbers of introns and the open reading frame (ORF) of the $m r-m a g R$ gene. The hydrophilicity, physical and chemical properties, transmembrane region,

${ }^{1}$ www.softberry.com signal peptide, subcellular localization, and secondary structure analysis of Mr-MagR were predicted and analyzed by the ExPASy-Prot Param tool and ExPASy-ProtScale (Gasteiger et al., 2005), TMHMM Server v. 2.02 and TMpred Server $^{3}$, SignalP 3.0 Server (Bendtsen et al., 2004), Psort II Prediction (Horton and Nakai, 1997), and PredictProtein and Sopma, respectively.

\section{Mr-MagR Heterologous Expression in E. coli \\ Construction of the Expression Vector for Mr-MagR}

After RNA was extracted from M7 mycelia grown on PDA for $96 \mathrm{~h}$ at $28^{\circ} \mathrm{C}$ according to the manufacturer's protocol (EZ-10 Spin Column RNA Purification Kit, BBI Markham, Canada), the full-length cDNA of $m r-m a g R$ was achieved according to the rapid amplification of cDNA end (RACE) method previously reported (Yang et al., 2012). The fulllength cDNA of $m r$-magR was sequenced (Sangon Biotech Co., Ltd., Shanghai, China), and its largest ORF was analyzed with ORF-Finder. After that, we designed the primers and the restriction sites with protective bases. Then the cDNA of M7 was used as a template to amplify $m r-m a g R$, and the final products were double-digested with corresponding restriction endonucleases and ligated to the pET-28a plasmid treated with the same digestion to get the expression vector. Finally, the expression vector (pET-28a-mr-magR) was transformed into E. coli $\mathrm{DH} 5 \alpha$ competent cells by a chemical transformation method for enrichment and preservation. The nested primers used in the RACE method and the primers used to construct the expression vector are shown in Table 1.

\footnotetext{
${ }^{2}$ http://www.cbs.dtu.dk/services/TMHMM/

${ }^{3}$ https://embnet.vital-it.ch/software/TMPRED_form.html
} 
TABLE 1 | Primers used for RACE method and construction of expression vectors.

\begin{tabular}{|c|c|c|}
\hline Primer names & Sequences $\left(5^{\prime}-3^{\prime}\right)$ & Functions \\
\hline $3^{\prime}$ connector 1 & 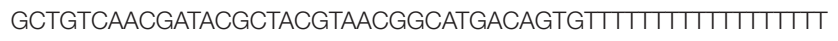 & 3'RACE first round of PCR amplification \\
\hline $3^{\prime}$ connector 2 & GATACGCTACGTAACGGCATGACAG & 3'second round of PCR amplification \\
\hline 5F02 & GACTCGAGTCGACATCGACCCCCCCCCCCCCCCCC & 5'RACE PCR amplification \\
\hline 5'Mr-MagR GSP01 & GCСTCTCССTCTCTCACGCTAA & 5'nested PCR amplification \\
\hline 5'Mr-MagR GSP02 & GGTAAGGTGTGAGGACGATAAG & $5^{\prime}$ nested PCR amplification \\
\hline 3'Mr-MagR GSP1 & TGGGTGTGAAGAATCGGGGCTG & 3'nested PCR amplification \\
\hline 3'Mr-MagR GSP2 & ATGGGTITACTTGGGGCGTCT & 3'nested PCR amplification \\
\hline Mr-MagR-His-up-BamHI & CGCGGATCCATGTCGTITGCTGCACCGTT & mr-magR 5'amplification \\
\hline Mr-MagR-His-do-Xhol & CCGCTCGAGAACCATGAAAGATTCACCGCATC & $m r-m a g R 3^{\prime}$ amplification \\
\hline
\end{tabular}

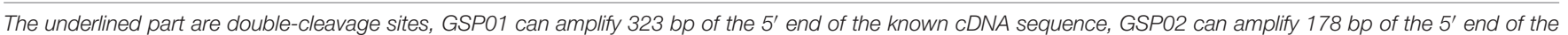
known cDNA sequence; GSP1 can amplify 332 bp 3' end of the known cDNA sequence, GSP2 can amplify 97 bp of the 3' end of the known cDNA sequence.

\section{Mr-MagR Expressed in E. coli}

The pET-28a-mr-magR plasmids were transformed into E. coli BL21 (DE3), and the expression strains were verified by colony PCR. The expression strains (pET-28a-mrmagR-BL21) were incubated in LB medium containing kanamycin. When $O D_{600 n m}$ value of the bacterial cells was up to about 0.6 , they were treated with 0 (CK), 0.1, $0.2, \quad 0.5,1,2 \mathrm{mM}$ isopropyl $\beta$-D-1-thiogalactopyranoside (IPTG) overnight at $15^{\circ} \mathrm{C}$ to explore the optimal inducer concentration. The cells were harvested by centrifugation at $12,000 \times \mathrm{g}, 4^{\circ} \mathrm{C}$, then resuspended in the lysis buffer (10 mM phosphate buffer saline (PBS), pH 7.5, $15 \mathrm{mM}$ dithiothreitol) containing a complete protease inhibitor cocktail (CWBIO Biotechnology Co., Ltd., Beijing, China) and lysed by sonication on ice. The supernatant and precipitation of the lysate were collected through centrifugation, and ran SDS-PAGE, respectively.

\section{Renaturation, Purification, and Western Blot Identification of Mr-MagR}

Because Mr-MagR was expressed in E. coli BL21 (DE3) as the inclusion body (see results), after the bacterial cells were harvested and resuspended in the lysis buffer on ice, the inclusion body was washed twice with the PBS, 1\% TritonX-100, $5 \mathrm{mM}$ EDTA ( $\mathrm{pH} 7.5$ ), resuspended in PBS ( $\mathrm{pH} 7.5)$ and washed to remove EDTA by centrifugation. The samples were diluted to the appropriate concentration $(10 \mathrm{~mL}$ lysis buffer per gram of wet cells) in $6 \mathrm{M}$ urea PBS to dissolve the precipitate, and we added $15 \mathrm{mM}$ dithiothreitol to open the disulfide bond for $1 \mathrm{~h}$ at $25^{\circ} \mathrm{C}$. Then, PBS with $0.3 \mathrm{mM}$ oxidized glutathione and $3 \mathrm{mM}$ reduced glutathione redox system (GSH-GSSG system) was configured as dialysate to promote the correct formation of disulfide bonds and to initiate the oxidative refolding. It should be noted that the appropriate volume of dialysate needs to be calculated to make the urea concentration equal to $4 \mathrm{M}$ before the first time changing the dialysate. Then, the dialysate should be renewed every $8 \mathrm{~h}$, and the final urea concentration should be $2 \mathrm{M}$ and $1 \mathrm{M}$ in order by calculating the volume added. During this process, the target protein $\mathrm{Mr}-\mathrm{MagR}$ was allowed slowly into a thermodynamically stable structure at $4^{\circ} \mathrm{C}$ and we got the soluble Mr-MagR protein.
The soluble Mr-MagR was purified with $\mathrm{Ni}^{2+}$-NTA (Sangon Biotech Co., Ltd., Shanghai, China), and ran SDS-PAGE. Then it was transferred to PVDF membrane to conduct Western blot identification (Shen et al., 2018).

\section{RESULTS}

\section{Effects of MF, BL, and MF-BL on Morphologies of $M$. ruber M7}

In order to investigate the effects of $\mathrm{MF}, \mathrm{BL}$, and $\mathrm{MF}-\mathrm{BL}$ on the growth of M7, we used different intensities of MF $(5,10$, and 30 $\mathrm{mT}$ ), 500 lux BL, and their combination (MF-BL) to, treat the M7 strain in the magnetic-light device, respectively (Figure 1).

The results of the 5 and $10 \mathrm{mT}$ conditions were almost same as $30 \mathrm{mT}$, so only $30 \mathrm{mT}$ pictures are shown here. The details of colonial and microscopic morphologies are described in Table 2 and Figure 2.

The results (Table 2 and Figure 2) showed that, compared with $\mathrm{CK}$, the different densities of MF tested in this study have no effect on the M7 growth, but 500 lux BL significantly inhibited the colonial growth of M7, under which the colonies looked like concentric circles with less and white aerial hyphae (Figure 2A). It is worth mentioning that, at 500 lux BL, small and immaturelike cleistothecia could be clearly observed only when M7 was cultured for 4 days; subsequently, the numbers of cleistothecia decreased significantly, and at 7 days, the cleistothecia could not be observed anymore (Figure $2 \mathbf{B}$ ).

Under MF-BL, however, the colonies were obviously bulging, volcanic-like with folds and a lot of white aerial hyphae, and the small and immature-like cleistothecia were observed throughout the culture period from 4 to 10 days, indicating that the MF-BL can restore the partial growth of M7 and its sexual generation, which were inhibited by 500 lux BL (Table 2 and Figure 2).

\section{Bioinformatics Investigation of Mr-MagR in M. ruber M7}

Based on the AA sequences of dMagR, we searched the genomes of animals, plants, and microorganisms, including Monascus spp., from NCBI databases using BLASTP and summarized a total of 73 proteins' AA sequences with a dMagR similarity 
TABLE 2 | Effects of 30 mT MF, 500 lux BL, and 30 mT-500 lux MF-BL on morphologies on M. ruber M7 (10 days).

\begin{tabular}{|c|c|c|c|c|c|}
\hline \multirow[t]{2}{*}{ Treatment } & \multicolumn{3}{|c|}{ Colonial characteristics } & \multirow{2}{*}{$\begin{array}{l}\text { Asexual } \\
\text { generation }\end{array}$} & \multirow[t]{2}{*}{ Sexual generation } \\
\hline & $\mathbf{M}\left(\Phi^{\star}\right) \pm \sigma$ & Mycelia & Colonial description & & \\
\hline $\mathrm{CK}^{\star \star}$ & $30 \pm 0.47$ & $\begin{array}{l}\text { Velvet-like, white aerial } \\
\text { hypha. }\end{array}$ & $\begin{array}{l}\text { The colony bulged with radiating } \\
\text { patterns and pink-red overall. Light pink } \\
\text { spores and aerial mycelia were } \\
\text { distributed throughout the colony. }\end{array}$ & Conidia in chains. & $\begin{array}{l}\text { Cleistothecia can be } \\
\text { formed, and they burst to } \\
\text { release ascospores. }\end{array}$ \\
\hline MF & $33 \pm 2.05$ & Same as CK. & Same as CK. & Same as CK. & Same as CK. \\
\hline$B L$ & $22 \pm 1.63^{*}$ & $\begin{array}{l}\text { Flocculent interlaced, } \\
\text { trace white aerial } \\
\text { mycelia. }\end{array}$ & $\begin{array}{l}\text { The colonies were slightly bulging, } \\
\text { orange overall, and have three } \\
\text { concentric circles: a large number of } \\
\text { white aerial mycelia and spores were } \\
\text { accumulated in the center area, and the } \\
\text { first circle near the center was } \\
\text { orange-yellow, light in color, and almost } \\
\text { no mycelia; the second circle was } \\
\text { formed almost entirely of white mycelia } \\
\text { and spores and was macroscopically } \\
\text { white; the outermost circle was darker } \\
\text { orange-red with jagged edges. }\end{array}$ & $\begin{array}{l}\text { A large number of } \\
\text { conidia in chains or } \\
\text { freely. }\end{array}$ & $\begin{array}{l}\text { Cleistothecia cannot be } \\
\text { formed after } 7 \text { days. }\end{array}$ \\
\hline MF-BL & $22 \pm 0.81^{\star}$ & $\begin{array}{l}\text { Many flocculent and } \\
\text { velvety white mycelia } \\
\text { were staggered. }\end{array}$ & $\begin{array}{l}\text { The colonies were volcanic-like highly } \\
\text { bulging, with a waxy edge and rough } \\
\text { surface with darker red, and a large } \\
\text { number of white aerial hyphae adhering } \\
\text { to the surface. }\end{array}$ & Same as CK. & Same as CK. \\
\hline
\end{tabular}

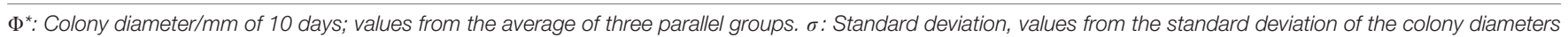
of the three parallel groups. ${ }^{*}$ Significantly different $(P<0.05)$. $\mathrm{CK}^{\star *}$ : Control check, in $28^{\circ} \mathrm{C}$, dark condition, neither BL nor MF.

greater than $55 \%$ and constructed a phylogenetic tree and analyzed AA sequences (Figure 3).

\section{Phylogenetic Analysis of Mr-MagR}

We find that MagR is ubiquitous in different species, and some organisms possess more than one MagR, indicating that the biological function of MagR may be highly conserved and indispensable in a wide range of organisms (Figure 3).

The MagR phylogenetic tree can be divided into three evolutionary branches as a whole when the bootstrap value is 1000. In Branch I, all MagRs are from bacteria. In Branch II, all MagRs are from animals, plants, and bacteria. In Branch III, MagRs come from animals, plants, fungi, and some bacteria, including MagRs from the Monascus species, which were clustered into a subcluster with $90 \%$ similarity and the closest to the MagR from Aspergillus terreus with 75\% similarity (Figure 3). It is very interesting that MagRs in the same species may belong to different branches, indicating that different MagRs in the same species may have different ancestry. For example, Glycine max possesses four homologous sequences of MagR: two of them are in Branch II, and the others are in Branch III.

A gene, GME5706 $\mathrm{g}$ in the M. ruber M7 genome, for which the $\mathrm{E}$ value is $7 \mathrm{E}^{-37}, 972 \mathrm{bp}$, containing two introns and three exons, with the highest match with $d m a g R$ was found, hereinafter named $m r$-magR ( $m a g R$ of $M$. ruber M7). The gene most similar to $m r-$ magr is TQB69303.1, which was identified as an ISC assembly protein in the $M$. purpureus 1596 genome. The conserved domain analysis has revealed that Mr-MagR is an ISC assembly accessory protein belonging to the IscA/HesB superfamily (Zheng et al.,
1998), and the AA sequence 142-244 is a highly conserved domain of IscA/HesB.

In Mr-MagR, especially 3 cysteine (Cys, 172, 236, 238) and 5 Glycine (Gly, 171, 174, 196, 211, 239), 1 tyrosine (Tyr, 177), 1 aspartic (Asp, 189, 214), 2 phenylalanine (Phe, 224, 242), 1 asparagine (Asn, 229), 1 proline (Pro, 230), and 1 serine (Ser, 241), are highly conserved with ones from other species (Figure 4). The highly conservative sites of Cys are mainly responsible for the assembly of iron-sulfur clusters (Mandilaras and Missirlis, 2012; Wang H. X. et al., 2016).

\section{Structure and Property Analysis of Mr-MagR}

We used different bioinformatics tools to further analyze the structure and property of Mr-MagR. The results (Table 3) show that $\mathrm{Mr}-\mathrm{MagR}$ is an unstable basic protein with $27.10 \mathrm{kDa}$ molecular weight and 10.05 of isoelectric point. And it is also a hydrophilic protein without a transmembrane region and signal peptide.

The secondary structure prediction of Mr-MagR shows that there are $24.59 \%$ of $\alpha$-helix structure, $13.11 \%$ of $\beta$-sheet, and $3.69 \% \beta$-turn structure, $58.61 \%$ random coil structure, including $8 \alpha$-helix, $8 \beta$-sheet, $4 \beta$-turn (Figure 5).

\section{Full-Length Clone and Heterologous Expression of $m r-m a g R$ cDNA Gene Clone and the Construction of Heterogeneous Expression Host}

We obtained untranslated DNA fragments: 127 bp at the $3^{\prime}$-end and $187 \mathrm{bp}$ at the $5^{\prime}$-end of $m r$-magR cDNA by two rounds of 


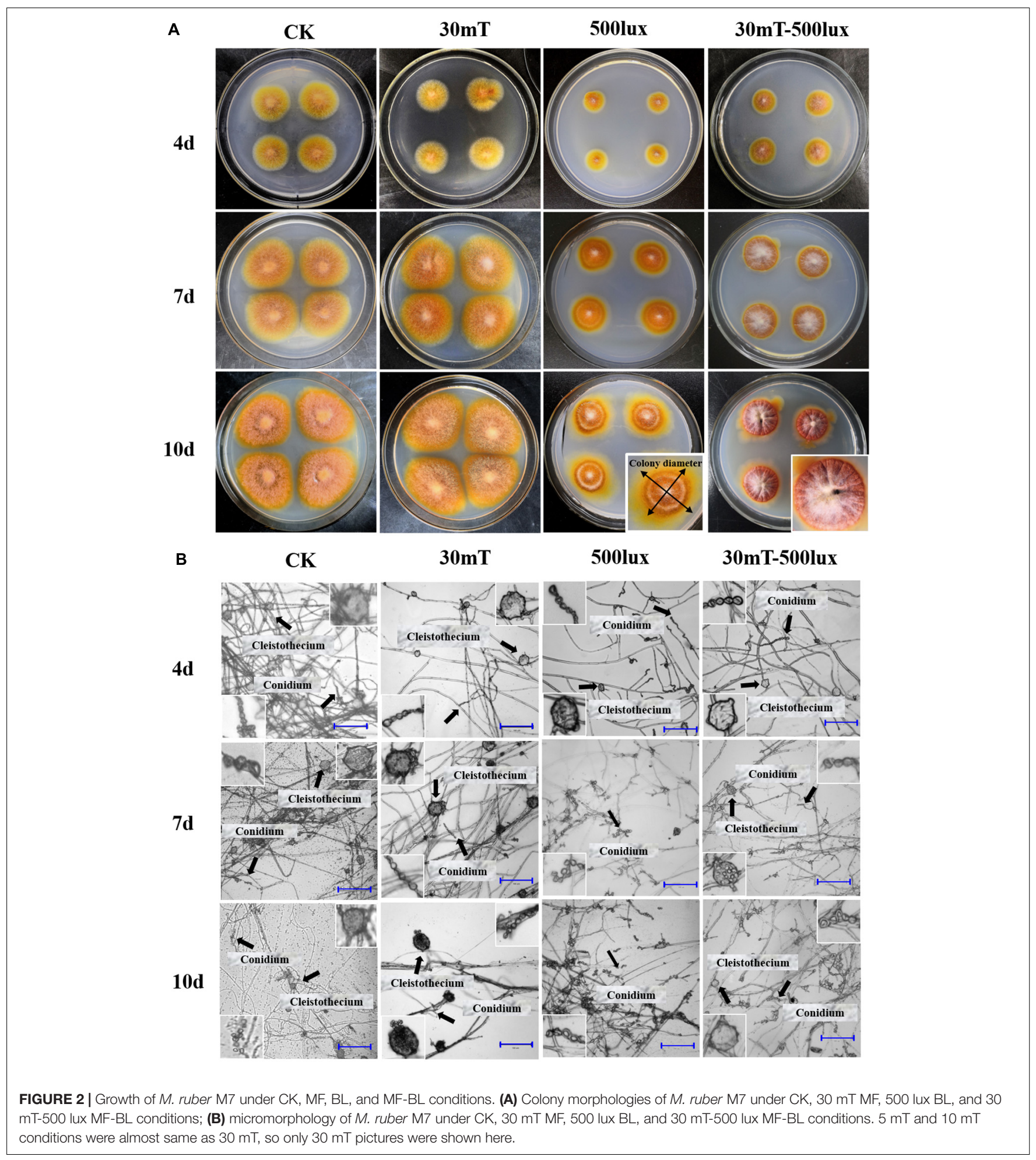

RACE-PCR based on the primers in Table 1. After adding the predicted $972 \mathrm{bp}$, we got the full-length cDNA of $m r$-magR with 1,286 bp. Then, we analyzed this 1286 bp by ORF finder, and the results show that the actual ORF of $m r$-magR is only $735 \mathrm{bp}$ (Figure 6B), which includes exon 1 (697 bp), exon 2 (31 bp), and the $3^{\prime}$ end ( $7 \mathrm{bp}$ ) of intron 2, indicating that intron 2 is not a true intron. The exon 3 was not included in the actual ORF due to the presence of the stop codon TGA at the $3^{\prime}$ end of intron 2 (Figure 6A). Therefore, the maximum ORF of $m r-m a g R$ is $735 \mathrm{bp}$, encoding $244 \mathrm{AA}$. 


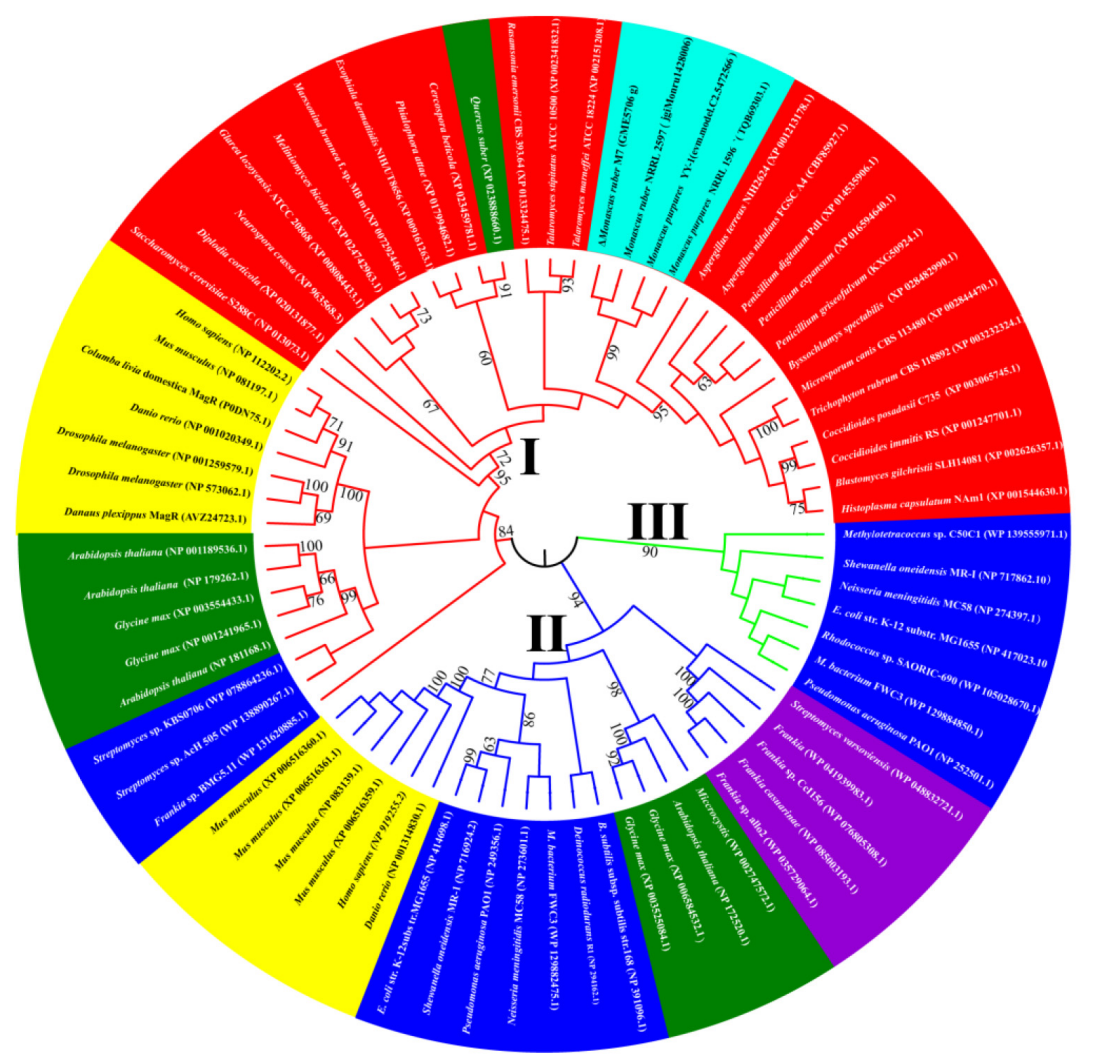

\section{Colored ranges}

$\square$ Animals
Plants
$\square$ Fungi
Bacteria
Actinomycetes
$\square$ Monascus spp.

FIGURE 3 | Phylogenetic trees of MagR. The AA sequence of the MagR of $D$. melanogaster published in NCBI as a control were aligned with the genome of four strains of Monascus spp. (M. ruber M7, M. ruber NRRL 2597, M. purpures YY-1, M. purpures NRRL 1596) and model organisms in animals, plants, and microorganisms in the Protein-NCBI database. A total of 73 MagRs with a homology $\geq 55 \%$ were selected and constructed with MEGA 7.0. The value next to the branch indicates the bootstrap support rate of the branch, and the bootstrap value was 1000 (Felsenstein, 1985). The evolution of Mr-MagR was estimated by the maximum likelihood method (Felsenstein, 1981). M. ruber M7 was marked by $\Delta$.

After the $m r-m a g R$ gene with 735 bp was cloned, its expression vector with His-tag, fused to the $\mathrm{N}$-terminal was constructed and expressed in E. coli BL21 (DE3).

\section{Expression, Renaturation, Purification, and Identification of Mr-MagR Protein}

After pET-28a-mr-magR-BL21 were incubated in LB medium containing kanamycin when the $O D_{600 \mathrm{~nm}}$ value of the bacterial cells was up to about 0.6 , the $E$. coli cells were induced with the $0.1 \mathrm{mM}$ IPTG, $15^{\circ} \mathrm{C}$ to produce $\mathrm{Mr}-\mathrm{MagR}$, which was mainly expressed in the inclusion body by running SDS-PAGE (Figure 7A). So the denaturing conditions were optimized, and 6 $\mathrm{M}$ urea-PBS was selected to dissolve Mr-MagR in the inclusion bodies. Finally, we got the target protein, after Mr-MagR was dialyzed in an improved GSH-GSSG system with $\mathrm{PBS}$ at $4^{\circ} \mathrm{C}$ for 24-36 h, purified with a Ni-NTA column (Figure 7B), detected by SDS-PAGE and identified by Western blot.

\section{DISCUSSION}

The behavior of many animals, such as migratory birds, monarch butterflies, salmon, and lobsters to orientate and migrate over long distances was discovered in the last century (Matthews, 1951; Quinn, 1980; Boles and Lohmann, 2003; Cain et al., 2005; Zhan et al., 2011). To elucidate this phenomenon, many hypotheses have been proposed, among which the magnetitebased model and RCM (the radical-pair-reaction-based chemical model) are in-depth studies (Frankel and Blakemore, 1980; Ritz et al., 2000; Hore and Mouritsen, 2016; Nordmann et al., 2017). The main issue that currently exists in the magnetitebased model is that no magnetic particle has been found up to now. About RCM, cryptochrome (Cry) is thought to be a magnetic receptor (Hore and Mouritsen, 2016), and in 2016, a MagR-Cry protein complex was discovered as a biocompass, which can perceive orientation and intensity of magnetic fields (MF) (Qin et al., 2016). Although such biocompass still has controversies, it may provide a new clue in this field. Especially, the MagR-Cry complex couples the effects of MF and light on organisms.

In this study, we found that the MF-BL has a significant effect on the growth of $M$. ruber M7 (Figure 2), suggesting that M7 may be able to perceive magnetic and light signals. We also searched the $m r-m a g R$ gene but did not find any gene homologous to the $\mathrm{BL}$ receptor including cry in the M7 genome (Wang L. et al., 2016). In order to explore if there 
Penicillium expansum (XP 016594640.1) Exophiala dermatitidis NIH/TU8656 (XP 09161263.1) Microsporum canis CBS 113480 (XP 002844470.1$)$ Meliniomyces bicolor E (XP 024742963.1) Coccidioides posadasii C735 (XP 003065745.1) Coccidioides immitis RS (XP 001247701.1) Trichophyton rubrum CBS 118932 (XP 003232324.1) Marssonina brunnea (XP 007292446.1) Blastomyces gilchristii SLH14081 (XP 002626357.1) Phialophora attae (XP 0117994682.1) Cercospora beticola (XP 023459781.1) Quercus suber (XP 023888660.1) Diplodia corticola (XP 020131877.1) Histoplasma capsulatum NAml (XP 001544630.1) Aspergillus terreus NIH2624 (XP 001213178.1) Aspergillus nidulans FGSC A4 (CBF 85927.1) Penicillium griseofulvum (XP 014535906.1) Penicillium griseofulvum (KXG 50924.1) AMonascus ruber M7 (GME 5706 g) Monascus ruber NRRL 1597 (jgi monrul 428006) Monascus purpures YY-1 (evm. model. C2. 5472566)
Monascus purpures NRRL 1596 (jgi monpul 501839) Monascus purpures NRRL 1596 (jgi
Neurospora crassa (XP 963568.3) Neurospora crassa (XP 963568.3)
Columba livia domestica MagR (PODN75.1) Danaus plexippus (AVZ24723.1) Saccharomyces cerevisiae S288C (NP 013073.1) Mus musculus (NP 081197.1) Homo sapiens (NP 112202.2)
Drosophila melanogaster (NP 573062.1) Drosophila melanogaster (NP Drosophila melanogaster (NP 001259579.1) Glycine max (XP 00355443.1) Arabidopsis thaliana (NP 179262.1) Dio rerio (NP 001020349.) Arabidopsis thaliana (NP 001189536.1) Arabidopsis thaliana (NP 181168.1) Shewanella oneidensis (NP 7417862.1) Pseudomonas aeruginosa (NP 274397.1)
E. coli str. K-12 substr. MG1655 (NP 417023.1) Glycine max (XP 006584532.1) Glycine max (XP 006584532.1) Arabidopsis thaliana (NP 172520.1) Arabidomsis thas aeruginosa PAO1 (NP 249356.1) Microcystis (WP 002747572.1)

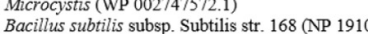
Shewanella oneidensis MR-1 (NP 716924.2) E. coli str. K-12 substr. MG1655 (NP 391096.1) Deinococcus radiodurans R1 (NP 294162.1) Deinococcus radiodulans (NP 294162.1) Neisseria meningitidis MCs8 (NP 273601.1) Deninococcus rodidis Neisseria meningitidis $\mathrm{MC} 58$ Homo sapiens (NP 919255.2) Mus musculus (XP 006516359.1) Mus musculus (NP 083139.1) Mus musculus (XP 006516361.1) Mus musculus (XP 006516360 1) Consensus

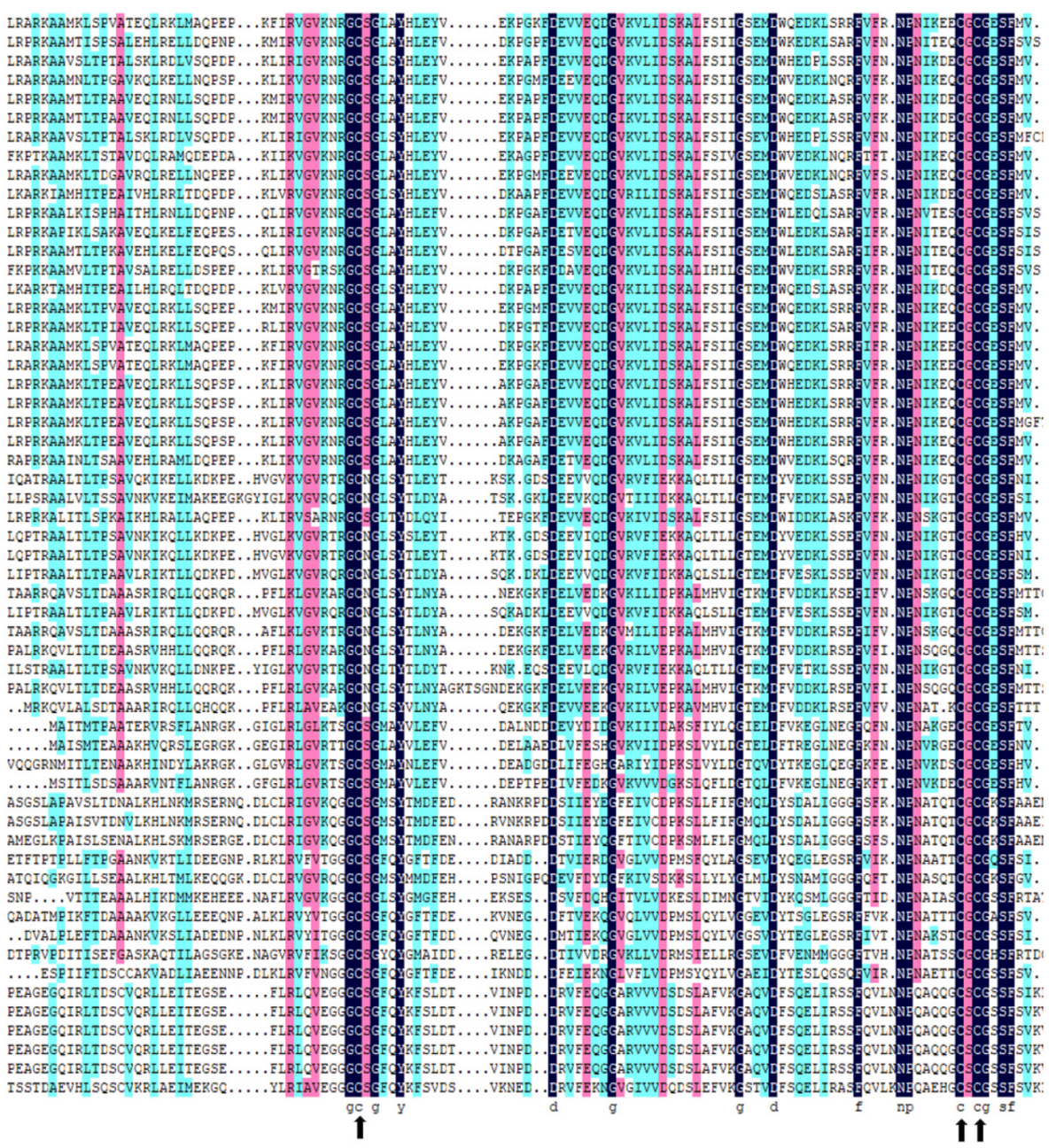

FIGURE 4 | Conservative analysis of AA sequence of Mr-MagR homologous gene. The AA homology is identified by different colors: black for 100\% homology, pink for $\geq 75 \%$, and bright blue for $\geq 50 \%$. Black arrows indicate highly conserved cysteine (Cys) residue positions.

TABLE 3 | Bioinformatics analysis of the AA sequence of Mr-MagR.

\begin{tabular}{|c|c|c|c|c|c|c|}
\hline AA No. & $\mathbf{M M}^{*}$ & $I^{* \star *}$ & Arg + Lys/Asp + Glu & IS index $x^{\star * *}$ & GRAVYT & $\mathrm{EPM}^{\star \star \star \star}$ \\
\hline 244 & 27106.00 & 10.05 & $38 / 21$ & 59.99 & -0.57 & $78.3 \%$ \\
\hline
\end{tabular}

$M^{*}$ : Protein molecular mass. IP**: Isoelectric point. IS index ${ }^{\star * *}$ : Protein instability index. EPM***: Probability of protein expression in mitochondria.

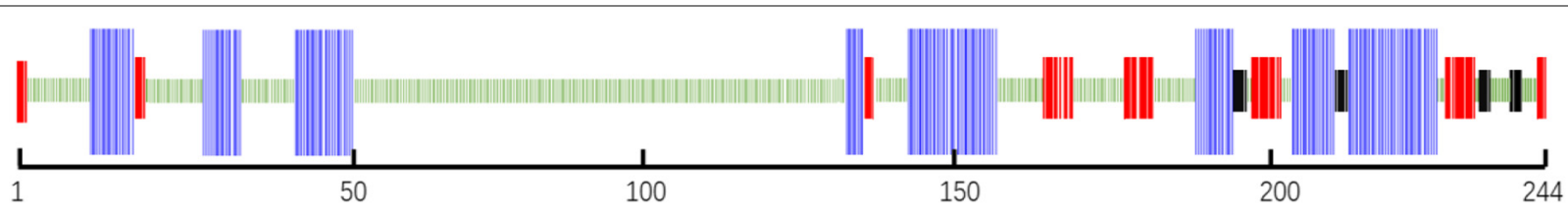

FIGURE 5 | Secondary structure analysis of Mr-MagR. The blue parts represent $8 \alpha$-helix structures, the red parts represent $8 \beta$-sheet structures, the black parts represent $4 \beta$-turn structures, and the remaining green parts are random coil structures.

exists any unknown BL receptor (Mr-BLR) in the M. ruber M7 genome and investigate interaction of Mr-MagR with Mr-BLR, we plan to use an improved His pull-down method to find the Mr-BLR from the whole M7 protein pool, which requires obtaining the Mr-MagR protein and using it to get the MrBLR protein through the protein-protein interaction method. So the results in this study will lay the foundation for finding a new BL receptor. 


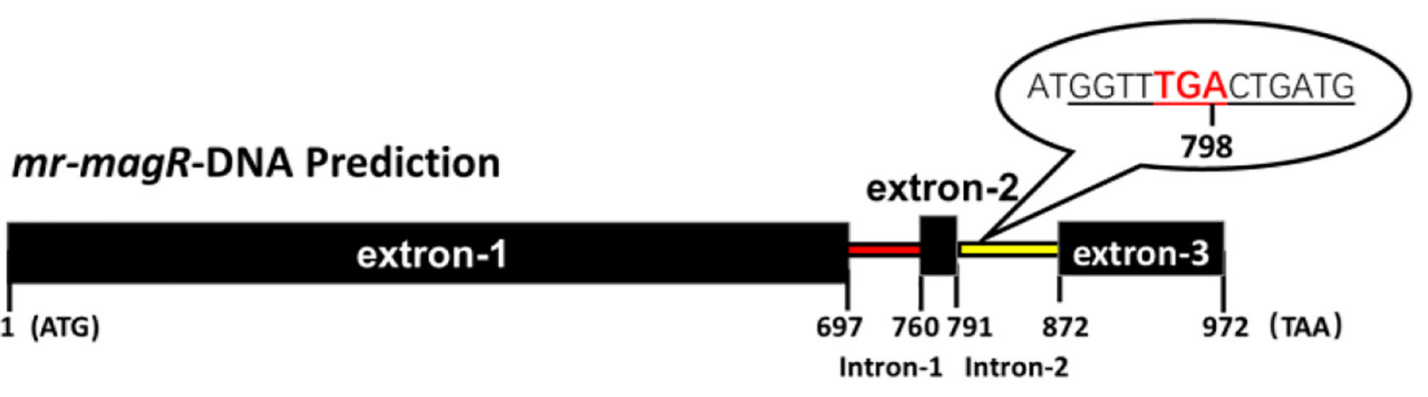

\section{RACE-mr-magR ORF}

B


FIGURE 6 | DNA, cDNA, and AA sequence information for mr-magR. (A) The prediction of the mr-magR gene is 972 bp, including two introns; the underlined part is the $5^{\prime}$-end partial sequence of intron 2; TGA is the stop codon. (B) mr-magR obtained by RACE-PCR amplification, and the ORF is 735 bp; (C) NCBI-CDD analysis shows that Mr-MagR belongs to the iron-sulfur cluster assembly accessory protein of the IscA/HesB superfamily, and the 142-244 AA are conserved domains.

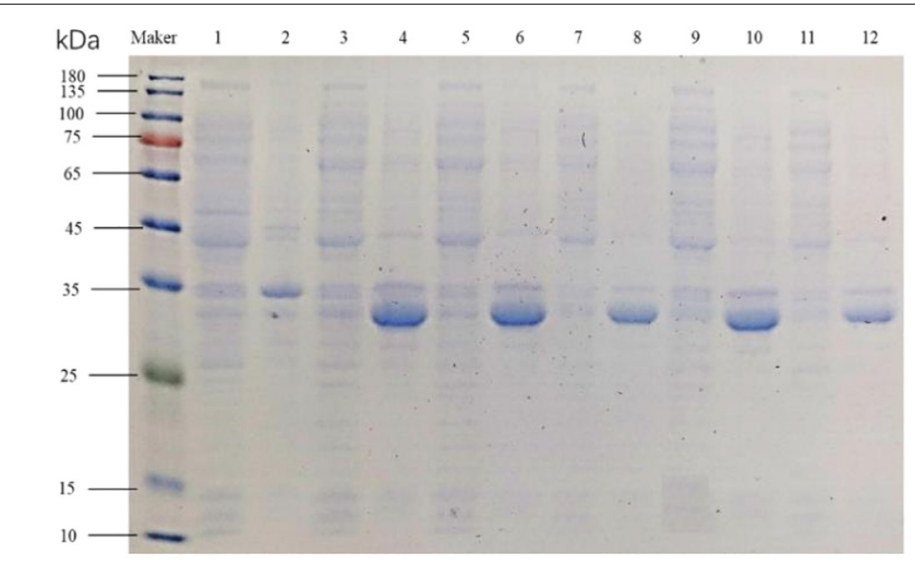

A

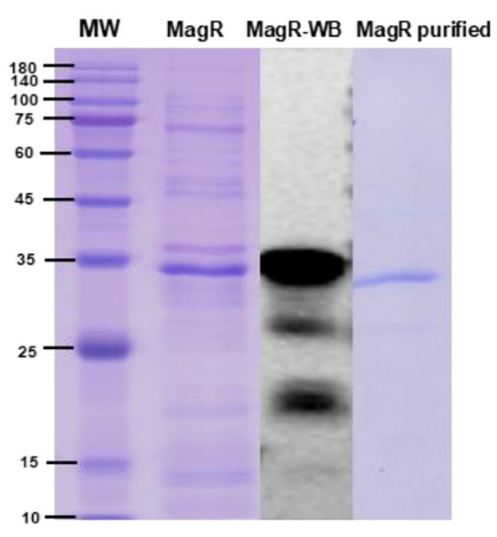

B

FIGURE 7 | Induced expression, purification, and WB verification of Mr-MagR protein. (A) 1: Supernatant without inducer; 2: Precipitate without inducer; 3, 4: Supernatant and precipitate of $0.1 \mathrm{mM} \mathrm{IPTG;} \mathrm{5,} \mathrm{6:} \mathrm{Supernatant} \mathrm{and} \mathrm{precipitate} \mathrm{of} \mathrm{0.2} \mathrm{mM} \mathrm{IPTG;} \mathrm{7,} \mathrm{8:} \mathrm{Supernatant} \mathrm{and} \mathrm{precipitate} \mathrm{of} \mathrm{0.5} \mathrm{mM} \mathrm{IPTG;} \mathrm{9,} \mathrm{10:}$ Supernatant and precipitate of 1 mM IPTG; 11, 12: Supernatant and precipitate of 2 mM IPTG. (B) Purification and WB verification results of Mr-MagR protein.

In current study, we have found that MF-BL can partially restore the growth and the sexual generation of M7, which are inhibited by 500 lux of BL although MF alone has no effect seemingly on M7 (Figure 2 and Table 2). We are doing more experiments to explain this result.
Bioinformatics analysis of $m r$-magR shows that it contains two introns (Figure 6A), but the full-length cDNA of $m r$ magR obtained by RACE-PCR showed that the gene only contains intron 1 (Figure 6B). Intron 2 may be involved in the expression of this gene through alternative splicing (Grützmann et al., 2013), which was common in eukaryotes. 
Therefore, we also designed primers and cloned and expressed the predicted $m r$-magR cDNA sequence in $E$. coli as well. The band size was the same as the protein expressed in this study. This result indicates that the $3^{\prime}$-end of intron 2 was indeed involved in gene transcription, confirming that it was indeed an exon. In order to initially explore the conditions for alternative splicing of this gene, we extracted total RNA from M7 at different culture times (3, 4, and 5 days) for RACE-PCR amplification, and the results showed that the gene does indeed only contain intron 1 . These results indicated that RACE-PCR was necessary for the heterologous expression of eukaryotic genes.

To get soluble target protein, we truncated expression of the highly conserved domain of $m r-m a g R$ ( $m r-c d m a g R$, AA NO.:142-244, see Figure 6C), to obtain the protein containing the core function of Mr-MagR. Then, we connected $m r-m a g R$ and $m r-c d m a g R$ to the pGEX-GST-TEV and pATX-SUMO plasmid, respectively, which are commonly used dissolution tags, to induce expression. Yet the protein still existed as an inclusion body. We also performed a heterologous expression of Mr-MagR in Pichai pastoris. Neither Mr-MagR nor MrCDMagR were expressed in $P$. pastoris, suggesting that directly obtaining soluble $\mathrm{Mr}-\mathrm{MagR}$ may require an expression system closer to the M. ruber M7, such as Aspergillus oryzae or Aspergillus niger.

\section{REFERENCES}

Anand, A., Nagarajan, S., Verma, A. P. S., Joshi, D. K., and Bhardwaj, J. (2012). Pre-treatment of seeds with static magnetic field ameliorates soil water stress in seedlings of maize (Zea mays L.). Indian J. Biochem. Biophys. 49, 63-70.

Bendtsen, J. D., Nielsen, H., von Heijne, G., and Brunak, S. (2004). Improved prediction of signal peptides: signalP 3.0. J. Mol. Biol. 340, 783-795. doi: 10. 1016/j.jmb.2004.05.028

Blakemore, R. (1975). Magnetotactic bacteria. Science 190, 377-379. doi: 10.1126/ science. 170679

Boles, L. C., and Lohmann, K. J. (2003). True navigation and magnetic maps in spiny lobsters. Nature 421, 60-63. doi: 10.1038/nature01226

Cain, S. D., Boles, L. C., Wang, J. H., and Lohmann, K. J. (2005). Magnetic orientation and navigation in marine turtles, lobsters, and molluscs: concepts and conundrums. Integrat. Comp. Biol. 45, 539-546. doi: 10.1093/icb/45. 3.539

Casas-Flores, S., and Herrera-Estrella, A. (2016). "3 the bright and dark sides of fungal life," in Environmental and Microbial Relationships, eds C. P. Kubicek and I. S. Druzhinina (Berlin: Springer), 41-77. doi: 10.1007/978-3-319-29532-9_3

Chen, F., and Hu, X. (2005). Study on red fermented rice with high concentration of monacolin $\mathrm{K}$ and low concentration of citrinin. Int. J. Food Microbiol. 103, 331-337. doi: 10.1016/j.ijfoodmicro.2005.03.002

Chen, W., Chen, R., Liu, Q., He, Y., He, K., Ding, X., et al. (2017). Orange, red, yellow: biosynthesis of azaphilone pigments in Monascus fungi. Chem. Sci. 8, 4917-4925. doi: 10.1039/C7SC00475C

Chen, W., He, Y., Zhou, Y., Shao, Y., Feng, Y., Li, M., et al. (2015). Edible filamentous fungi from the species Monascus: early traditional fermentations, modern molecular biology, and future genomics. Compr. Rev. Food Sci. Food Saf. 14, 555-567. doi: 10.1111/1541-4337.12145

Ding, B., Smith, E. S., and Ding, H. (2005). Mobilization of the iron centre in IscA for the iron-sulphur cluster assembly in IscU. Biochem. J. 389, 797-802. doi: 10.1042/BJ20050405

El-Gebali, S., Mistry, J., Bateman, A., Eddy, S. R., Luciani, A., Potter, S. C., et al. (2018). The Pfam protein families database in 2019. Nucleic Acids Res. 47, D427-D432. doi: 10.1093/nar/gky995

Faivre, D., and Schuler, D. (2008). Magnetotactic bacteria and magnetosomes. Chem. Rev. 108, 4875-4898. doi: 10.1021/cr078258w

\section{DATA AVAILABILITY STATEMENT}

The raw data supporting the conclusions of this article will be made available by the authors, without undue reservation, to any qualified researcher.

\section{AUTHOR CONTRIBUTIONS}

FC supervised the entire work, planned the experiments, and revised the manuscript. HZ performed the experiments, analyzed the data, and wrote the manuscript. SY guided HZ to conduct the experiments in molecular biology parts. All authors contributed to the manuscript and approved the submitted version.

\section{FUNDING}

This work was supported by the National Key Research and Development Program of China (No. 2018YFD0400404), the Major Program of the National Natural Science Foundation of China (Nos. 31730068 and 31330059), and the Shandong Province Taishan Industry Leading Talents High-Efficiency Agriculture Innovation Project (No. tscy20180120).

Felsenstein, J. (1981). Evolutionary trees from DNA sequences: a maximum likelihood approach. J. Mol. Evol. 17, 368-376. doi: 10.1007/BF017 34359

Felsenstein, J. (1985). Confidence limits on phylogenies: an approach using the bootstrap. Evolution 39, 783-791. doi: 10.1111/j.1558-5646.1985.tb00420.x

Frankel, R. B., and Blakemore, R. P. (1980). Navigational compass in magnetic bacteria. J. Magn. Magn. Mater. 1, 1562-1564. doi: 10.1016/0304-8853(80) 90409-6

Frankel, R. B., Blakemore, R. P., and Wolfe, R. S. (1979). Magnetite in freshwater magnetotactic bacteria. Science 203, 1355-1356. doi: 10.1126/science.203.4387. 1355

Garcia, R. F., and Arza, P. L. (2001). Influence of a stationary magnetic field on water relations in lettuce seeds. Bioelectromagnetics 22, 589-595. doi: 10.1002/ bem. 89

Gasteiger, E., Hoogland, C., Gattiker, A., Wilkins, M. R., Appel, R. D., and Bairoch, A. (2005). "'Protein identification and analysis tools on the ExPASy server,", in The Proteomics Protocols Handbook, ed. J. M. Walker (Berlin: Springer), 571-607. doi: 10.1385/1-59259-890-0:571

Gegear, R. J., Casselman, A., Waddell, S., and Reppert, S. M. (2008). Cryptochrome mediates light-dependent magnetosensitivity in Drosophila. Nature 454, 10141018. doi: $10.1038 /$ nature 07183

Giachello, C. N. G., Scrutton, N. S., Jones, A. R., and Baines, R. A. (2016) Magnetic fields modulate blue-light-dependent regulation of neuronal firing by cryptochrome. J. Neurosci. 36, 10742-10749. doi: 10.1523/JNEUROSCI.214016.2016

Grützmann, K., Szafranski, K., Pohl, M., Voigt, K., Petzold, A., and Schuster, S. (2013). Fungal alternative splicing is associated with multicellular complexity and virulence: a genome-wide multi-species study. DNA Res. 21, 27-39. doi: 10.1093/dnares/dst038

Hore, P., and Mouritsen, H. (2016). The radical-pair mechanism of magnetoreception. Annu. Rev. Biophys. 45, 299-344. doi: 10.1146/annurevbiophys-032116-094545

Horton, P., and Nakai, K. (1997). Better Prediction of Protein Cellular Localization Sites with the it k Nearest Neighbors Classifier. Ismb 5, 147-152.

Jensen, L. T., and Culotta, V. C. (2000). Role of Saccharomyces cerevisiae ISAland ISA2 in iron homeostasis. Mol. Cell. Biol. 20, 3918-3927. doi: 10.1128/MCB.20. 11.3918-3927.2000 
Johnsen, S., and Lohmann, K. J. (2005). The physics and neurobiology of magnetoreception. Nat. Rev. Neurosci. 6, 703-712. doi: 10.1038/nrn1745

Kaut, A., Lange, H., Diekert, K., Kispal, G., and Lill, R. (2000). Isalp is a component of the mitochondrial machinery for maturation of cellular iron-sulfur proteins and requires conserved cysteine residues for function. J. Biol. Chem. 275, 15955-15961. doi: 10.1074/jbc.M909502199

Kosmidis, S., Botella, J. A., Mandilaras, K., Schneuwly, S., Skoulakis, E. M., Rouault, T. A., et al. (2011). Ferritin overexpression in Drosophila glia leads to iron deposition in the optic lobes and late-onset behavioral defects. Neurobiol. Dis. 43, 213-219. doi: 10.1016/j.nbd.2011. 03.013

Krebs, C., Agar, J. N., Smith, A. D., Frazzon, J., Dean, D. R., Huynh, B. H., et al. (2001). IscA, an alternate scaffold for Fe-S cluster biosynthesis. Biochemistry 40, 14069-14080. doi: 10.1021/bi015656z

Kumar, S., Stecher, G., and Tamura, K. (2016). MEGA7: molecular evolutionary genetics analysis version 7.0 for bigger datasets. Mol. Biol. Evol. 33, 1870-1874. doi: $10.1093 / \mathrm{molbev} / \mathrm{msw} 054$

Letunic, I., and Bork, P. (2019). Interactive Tree Of Life (iTOL) v4: recent updates and new developments. Nucleic Acids Res. 47, W256-W259. doi: 10.1093/nar/ gkz239

Liboff, A., Smith, S., and McLeod, B. (1987). "Experimental evidence for ion cyclotron resonance mediation of membrane transport," in Mechanistic Approaches to Interactions of Electric and Electromagnetic Fields With Living Systems, eds M. Blank and E. Findl (Berlin: Springer), 109-132. doi: 10.1007/ 978-1-4899-1968-7_7

Maeda, K., Henbest, K. B., Cintolesi, F., Kuprov, I., Rodgers, C. T., Liddell, P. A., et al. (2008). Chemical compass model of avian magnetoreception. Nature 453, 387-390. doi: 10.1038 /nature06834

Mandilaras, K., and Missirlis, F. (2012). Genes for iron metabolism influence circadian rhythms in Drosophila melanogaster. Metallomics 4, 928-936. doi: 10.1039/C2MT20065A

Marchler-Bauer, A., Bo, Y., Han, L., He, J., Lanczycki, C. J., Lu, S., et al. (2016). CDD/SPARCLE: functional classification of proteins via subfamily domain architectures. Nucleic Acids Res. 45, D200-D203. doi: 10.1093/nar/gkw1129

Matthews, G. T. (1951). The experimental investigation of navigation in homing pigeons. J. Exp. Biol. 28, 508-536.

Mouritsen, H., and Hore, P. (2012). The magnetic retina: light-dependent and trigeminal magnetoreception in migratory birds. Curr. Opin. Neurobiol. 22, 343-352. doi: 10.1016/j.conb.2012.01.005

Mouritsen, H., and Ritz, T. (2005). Magnetoreception and its use in bird navigation. Curr. Opin. Neurobiol. 15, 406-414. doi: 10.1016/j.conb.2005.06.003

Nordmann, G. C., Tobias, H., and Keays, D. A. (2017). Magnetoreception-A sense without a receptor. PLos Biol. 5:e2003234. doi: 10.1371/journal.pbio.2003234

O'Neill, P. (2013). Magnetoreception and baroreception in birds. Dev. Growth Differ. 55, 188-197. doi: 10.1111/dgd.12025

Pelzer, W., Mühlenhoff, U., Diekert, K., Siegmund, K., Kispal, G., and Lill, R. (2000). Mitochondrial Isa2p plays a crucial role in the maturation of cellular iron-sulfur proteins. FEBS Lett. 476, 134-139. doi: 10.1016/S0014-5793(00) 01711-7

Qin, S., Yin, H., Yang, C., Dou, Y., Liu, Z., Zhang, P., et al. (2016). A magnetic protein biocompass. Nat. Mater. 15, 217-226. doi: 10.1038/nmat4484

Quinn, T. P. (1980). Evidence for celestial and magnetic compass orientation in lake migrating sockeye salmon fry. J. Comp. Physiol. 137, 243-248. doi: 10.1007/ BF00657119

Rakoczy, R., Konopacki, M., and Fijałkowski, K. (2016). The influence of a ferrofluid in the presence of an external rotating magnetic field on the growth rate and cell metabolic activity of a wine yeast strain. Biochem. Eng. J. 109, 43-50. doi: 10.1016/j.bej.2016. 01.002
Ritz, T., Adem, S., and Schulten, K. (2000). A model for photoreceptor-based magnetoreception in birds. Biophys. J. 78, 707-718. doi: 10.1016/S00063495(00)76629-X

Ritz, T., Thalau, P., Phillips, J. B., Wiltschko, R., and Wiltschko, W. (2004). Resonance effects indicate a radical-pair mechanism for avian magnetic compass. Nature 429, 177-180 . doi: 10.1038/nature02534

Rodgers, C. T., and Hore, P. J. (2009). Chemical magnetoreception in birds: the radical pair mechanism. Proc. Natl. Acad. Sci. U.S.A. 106, 353-360. doi: 10.1073/ pnas. 0711968106

Schumacher, J. (2017). How light affects the life of Botrytis. Fungal Genet. Biol. 106, 26-41. doi: 10.1016/j.fgb.2017.06.002

Shen, Y., Wang, Y., Jiang, X., Lu, L., Wang, C., Luo, W., et al. (2018). Preparation and characterization of a high-affinity monoclonal antibody against human epididymis protein-4. Protein Exp. Purificat. 141, 44-51. doi: 10.1016/j.pep. 2017.09.005

Sherrard, R. M., Morellini, N., Jourdan, N., El-Esawi, M., Arthaut, L.-D., Niessner, C., et al. (2018). Low-intensity electromagnetic fields induce human cryptochrome to modulate intracellular reactive oxygen species. PLoS Biol. 16:e2006229. doi: 10.1371/journal.pbio.2006229

Wan, Y., Zhang, J., Han, H., Li, L., Liu, Y., and Gao, M. (2017). Citrinin-producing capacity of Monascus purpureus in response to low-frequency magnetic fields. Process Biochem. 53, 25-29. doi: 10.1016/j.procbio.2016.11.009

Wang, H. X., Xiang, Y. C., and Zhang, Y. G. (2016). The proceedings of Isca that functions as a biomagnetic receptor protein (MagR). Prog. Biochem. Biophys. 43, 1115-1128. doi: 10.16476/j.pibb.2016.0190

Wang, L., Dai, Y., Chen, W., Shao, Y., and Chen, F. (2016). Effects of light intensity and color on the biomass, extracellular red pigment, and citrinin production of Monascus ruber. J. Agric. Food Chem. 64, 9506-9514. doi: 10.1021/acs.jafc. $6 \mathrm{~b} 04056$

Wiltschko, R. (2012). Magnetic Orientation in Animals. Berlin: Springer Science \& Business Media.

Yang, Y., Li, L., Li, X., Shao, Y., and Chen, F. (2012). mrflbA, encoding a putative FlbA, is involved in aerial hyphal development and secondary metabolite production in Monascus ruber M-7. Fungal Biol. 116, 225-233. doi: 10.1016/ j.funbio.2011.11.005

Zhadin, M. N. (2001). Review of Russian literature on biological action of DC and low-frequency AC magnetic fields. Bioelectromagnetics 22, 27-45. doi: 10.1002/1521-186x(200101)22:1<27::aid-bem4>3.0.co;2-2

Zhan, S., Merlin, C., Boore, J. L., and Reppert, S. M. (2011). The monarch butterfly genome yields insights into long-distance migration. Cell 147, 1171-1185. doi: 10.1016/j.cell.2011.09.052

Zhang, J., Zeng, D., Xu, C., and Gao, M. (2015). Effect of low-frequency magnetic field on formation of pigments of Monascus purpureus. Eur. Food Res. Technol. 240, 577-582. doi: 10.1007/s00217-014-2358-x

Zheng, L., Cash, V. L., Flint, D. H., and Dean, D. R. (1998). Assembly of iron-sulfur clusters identification of an iscSUA-hscBA-fdx gene cluster from Azotobacter vinelandii. J. Biol. Chem. 273, 13264-13272. doi: 10.1074/jbc.273.21. 13264

Conflict of Interest: The authors declare that the research was conducted in the absence of any commercial or financial relationships that could be construed as a potential conflict of interest.

Copyright (c) 2020 Zhou, Yang and Chen. This is an open-access article distributed under the terms of the Creative Commons Attribution License (CC BY). The use, distribution or reproduction in other forums is permitted, provided the original author(s) and the copyright owner(s) are credited and that the original publication in this journal is cited, in accordance with accepted academic practice. No use, distribution or reproduction is permitted which does not comply with these terms. 